\title{
DIVISION IX / COMMISSION 25 / WORKING GROUP INFRARED ASTRONOMY
}

\author{
PRESIDENT \\ VICE-PRESIDENT \\ MEMBERS
}

\author{
Eugene F. Milone \\ Andrew T. Young \\ Roger A. Bell, Michael Bessell, \\ Richard P. Boyl, Martin Cohen, \\ David J.I. Fry, Robert Garrison, \\ Robert Garrison, Ian S. Glass \\ John Graham, Anahi Granada, \\ Lynn Hillenbrand, Robert L. Kurucz, \\ Ian McLean, \\ Matthew Mountain, George Riecke, \\ Rogerio Riffel, Ronald G. Samec, \\ Stephen J. Schiller, Douglas Simons, \\ Michael Skrutskie, C. Russell Stagg, \\ Christiaan L. Sterken, Roger I. Thompson, \\ Alan Tokunaga, Kevin Volk, Robert Wing.
}

\section{PROCEEDINGS BUSINESS SESSIONS, 7 August 2009}

\section{Introduction}

The formal origin of the IRWG occured at the Buenos Aires General Assembly, following a Joint Commission meeting at the IAU GA in Baltimore in 1988 that identified the problems with ground-based infrared photometry. The situation is summarized in Milone (1989). In short, the challenges involved how to explain the failure to achieve the milli-magnitude precision expected of infrared photometry and an apparent $3 \%$ limit on system transformability. The proposed solution was to redefine the broadband Johnson system, the passbands of which had proven so unsatisfactory that over time effectively different systems proliferated, although bearing the same $J H K L M N Q$ designations; the new system needed to be better positioned and centered in the atmospheric windows of the Earth's atmosphere, and the variable water vapour content of the atmosphere needed to be measured in real time to better correct for atmospheric extinction.

The IRWG established criteria for judging the performance of existing infrared passbands and experimented with passband shapes, widths, and placements within the spectral windows of the Earth's atmosphere. The method and coding were initiated and largely carried out by A. T. Young, and, aided by C. R. Stagg, Milone ran the simulations. The full details of the criteria and results of the numerical simulations were presented by Young et al. (1994). Subsequent work, described in WG-IR and/or Commission 25 reports, included the use of a newer MODTRAN version (3.7) to check and extend previous work. This part of the program proved so successful in minimizing the effects of water vapour on the source flux transmitted through the passband that the second stage, real-time monitoring of IR extinction, was not pursued, although this procedure remains desirable for unoptimized passbands designed for specific astrophysical purposes.

During the following triennia, the WG concentrated on gathering and presenting evidence of the usefulness of the IRWG infrared passband set. For the near infrared portion of the IRWG set (namely, the $i z, i J, i H, i K$ passbands), field trials were conducted over the years 1999-2003 with an InSb detector in a Dewar mounted on the 1.8-m telescope at the Rothney Astrophysical Observatory of the University of Calgary. The results of those trials and the details of further 
work were presented in Milone \& Young (2005). This paper contained, for the first time, evidence that not only were the IRWG passbands more useful to secure precise transformations than all previous passbands, but that they were also superior in at least one measure of the signal to noise ratio. This evidence was further refined in Milone \& Young (2007). As a consequence, the original purpose of the IRWG largely has been achieved, but resistance to the new passband system is still strong, and passbands that somewhat compromise the IRWG recommendations have been advanced in order to provide more throughput, at the cost of precision and standardization. Thus, nonoptimized passbands are still in use at the highest altitude infrared sites. The situation is described (and decried!) in Milone \& Young (2007). It would be incorrect to conclude, however, that the community has ignored the work of the IRWG. As noted in previous IRWG reports, there is now a general acceptance of the principles enunciated in Young et al. (1994), and incremental movement is occurring. However, the sharp break from the nomenclature of the Johnson passbands has not not been accepted, as the papers below illustrate. Many Infrared astronomers continue to use " $J H K L$ " designations even though there is demonstrably no single passband system with those designations. The IRWG suggests that the designations be assigned instead to the atmospheric windows most prominently associated with the original Johnson passbands, as done in Milone \& Young (2005). The Mauna Kea near-IR suite of passbands that has been described by Tokunaga \& Vacca (2007), is one of the best of the incrementally improved systems, but falls short of the IRWG specifications.

\section{IRWG Science and Business Session Meeting}

The meeting of the WG in the Rio de Janeiro GA was held during Session 2 on Aug. 7, 2009, and was chaired by Milone. The history of the IRWG was summarized, and an invitation for all photometrists with any interest in the IR to join was issued. The IRWG has had since its inception an open policy of membership, and this invitation was extended at the present GA.

Recent highlights of the work of the IRWG were described. In Milone \& Young (2005) and further in Milone \& Young (2007), correlations were seen among: our figure of merit, $\theta$, a measure of the distortion of the spectral irradiance of starlight as it descends the Earth's atmosphere; a measure of the Forbes effect (the rapid change in slope of the extinction curve with decreasing airmass); the extinction coefficient between 1 and higher airmasses; and a measure of the signal to noise ratio. Perhaps the most useful contribution in the past triennium was given in Milone \& Young (2008), which argues for the suitability of the IRWG passbands to provide millimagnitude precision for variable star infrared photometry, and that this is possible at ANY photometric site irrespective of its elevation. Directed to both professional and amateur astronomical communities, this paper compares extinction coefficients obtained using a sample of old IR filters with those using the IRWG passbands determined from the same night at the RAO. The coefficients for the old passbands are seen to be greater by factors $\sim 2$ or more, as predicted by the simulations and numerical experiments. The very small Forbes effect seen with the IRWG passbands, permits the use of the Bouguer extinction coefficients to obtain more accurate outside-atmosphere magnitudes than is possible with others, for which the Forbes effect can be very great.

A similar theme was emphasized in a column in the General Assembly's daily newspaper, Estrela D'alva, Day 10, p.4. Such a development is now possible thanks to bulk prices (for lots of 9 or greater) for the IRWG passbands by Custom Scientific, Inc. of Phoenix, Arizona, so far the only filter manufacturer who has produced these filters to our specifications. It is to be hoped that photometer manufacturers will offer installation of the IRWG iz, and iH filters, at least, in IR instruments that they sell, in place of the current filters that are not optimum.

\section{Other New Developments in IR Astronomy}

Among new work carried out during the past triennium is the following:

- Hora, et al. (2008) discuss the response functions of the passbands of the Spitzer Infrared Array Camera, and provide corrections, which, when applied, can lead to relative accuracy of $\sim 2 \%$;

- Monson \& Pierce (2009) discuss the performance of the BIRCAM array camera of the Wyoming Red Buttes Observatory, where it is mounted on a 24-in telescope. They equip this instrument with " $J H K$ " filters. 
- Sánchez, et al. $(2007,2008)$ discuss the infrared properties of their site, especially the extinction (in the first paper) and determine the sky brightness (in the second) in "JHK$K_{s}$ " passbands for all times of the year at the Calar Alto Observatory in Spain. They find strong variations in sky brightness, with the maximum in the summer, when the temperature (and humidity) is the highest.

- Taylor $(2007$; 2008a, 2008b) discusses observations made with the Wampler Scanner, including the extinction due to aerosols as well as water vapor.

- van Dokkum, et al. (2009) used three overlapping " $J$ " passbands and two " $H$ " passbands to locate the Balmer jump \& $400 \mathrm{~nm}$ break in galaxies over the $\mathrm{z}$ red shift range 1.5 to 3.5 and compare their passbands to the atmospheric transmission curve. The breaking of the conventional passbands into shorter segments, at least in part to improve transmission through the atmosphere, is a welcome step, even if it is not the main purpose of the work.

- Wood-Vasey, et al. (2008b), who use " $J H$ " and " $K s$ " passbands to demonstrate that SN Ia are standard candles. This paper demonstrates that infrared astronomy continues to be a very important tool for distance-scale work.

The wide use of $J H K$ designations for what are clearly not original Johnson passbands highlights the problem that infrared astronomers have been loath to face: There are no standard $J H K L$ passbands, notwithstanding the increasing use of "short" $K$ passbands (" $K_{s}$ ") that cut off the longer-wavelength end of the window, and thus cut down the thermal emission in the passband. The Mauna Kea near-infrared suite, an incremental improvement over older passbands sets, although falling short of the IRWG prescription, typifies the movement of infrared astronomy toward the goals of the WG.

\section{Discussion}

Several topics were put up for consideration and discussion during the scientific and business session. Among them were:

- Virtual Observatory Contributions;

- Update website content;

- Facilitate manufacture of IRWG filters;

- Extension of standard stars list (for Comm. 25 site);

- Testing of $i L, i L^{\prime}, i M, i N$, in, $i Q$ passbands;

- Real-time monitoring of $\mathrm{H}_{2} \mathrm{O}$ atmospheric content; and

- correlation with extinction effects.

Several of these topics had been discussed recently in Milone \& Young (2008). As noted already, IRWG filters are now available at bulk prices from Custom Scientific, Inc., of Phoenix, AZ. The prices may be obtained from that company. Milone expressed willingness to coordinate a bulk purchase, and urged those interested to contact him. The extension of standard stars lists for the Commission 25 website is dependent on the facilities of the former president of Comm. 25, Peter Martinez, who, however, has expressed interest in maintaining the website.

\section{Closing remarks}

The IRWG has now been in existence for two decades. Although there appeared to be lively interest in the work of the IRWG by those who attended the session, and depite incremental improvements in other IR passbands, it is clear that more work needs to be done to promote the IRWG passbands, especially for their use in variable star light curve acquisition. Astronomers are a conservative lot, and even though there is no standard JHKLMNQ system to which they need to be loyal, infrared astronomers have been particularly reluctant to adopt and try new filters, except to isolate particular spectral features. In most cases they may not want to sacrifice white-light filters (which is what conventional infrared filters have been, effectively) for narrower ones that provide less overall throughput, but are defined by the edges of the atmospheric windows. Such an attitude is followed by those operating in what can be called a "discovery" mode. Photometrists have basically different aims. One legitimate concern is that data taken in other IR passbands may not transform to the IRWG system, with the possible exception of data in the newer Mauna Kea set used at the one site in the world where it is most suitable, and when conditions there are dry. Consequently, it seems that only demonstrations of the superiority of the IRWG set on specific targets will convince many to use these filters. 
Therefore, we urge photometrists with a strong interest in precise photometry to give these passbands a try at observatories where photometry is done either with a chopping secondary and LIA system, as at the RAO, or with array cameras.

Eugene F. Milone Chair of the Working Group

\section{References}

van Dokkum, P. G., et al. 2009, PASP, 121, 2-8

Hora, J. L., et al. 2008, PASP, 120, 1233-1243

Milone 1989, in: E. F. Milone (ed.), Infrared Extinction and Standardization, Proc., Two Sessions of IAU Commissions 25 and 9, Baltimore, MD, USA, 4 August 1988, Lecture Notes in Physics, Vol. 341 (Heidelberg: Springer), p. 1

Milone, E. F. \& Young, A. T. 2005, PASP, 117, 485

Milone, E. F. \& Young, A. T. 2007, in: C. Sterken (ed.), The Future of Photometric, Spectrophotometric, and Polarimetric Standardization, Proc. Intern. Workhop, Blankenberge, Belgium, 8-11 May 2006, ASP-CS, 364, 387

Milone, E. F. \& Young, A. T. 2008, JRASC, 36, 110

Monson, A. J. \& Pierce, M. J. 2009, PASP, 121, 728

Sánchez, S. F., et al. 2007, PASP, 120, 1244

Sánchez, S. F., et al. 2008, PASP, 120, 1244

Taylor, B. J. 2007, PASP, 119, 407

Taylor, B. J. 2008, PASP, 120, 602

Taylor, B. J. 2008b, PASP, 120, 1183

Tokunaga, A. T. \& Vacca, W. D. 2007, in: C. Sterken (ed.), The Future of Photometric, Spectrophotometric, and Polarimetric Standardization, Proc. Intern. Workhop, Blankenberge, Belgium, 8-11 May 2006, ASP-CS, 364, 409

Wood-Vasey, W. M., et al. 2008, ApJ, 689, 377

Young, A. T., Milone, E. F., \& Stagg, C. R. 1994, A\&AS, 105, 259 